Pacific Journal of Mathematics

CORRESPONDING RESIDUE SYSTEMS IN ALGEBRAIC

HUB 


\title{
CORRESPONDING RESIDUE SYSTEMS IN ALGEBRAIC NUMBER FIELDS
}

\author{
H. S. Butts AND H. B. MANN
}

In this paper we shall consider integral ideals in finite algebraic extensions of the field $R$ of rational numbers. Algebraic number fields will be denoted by $\mathfrak{F}$ with subscripts or superscripts, ideals by German letters, algebraic numbers by lower case Greek letters, and numbers of the rational field $R$ by lower case Latin letters.

Two ideals in the same field are equal if and only if they contain the same numbers.

If $a_{1}$ is an ideal in a field $\widetilde{F}_{1}$ and $\mathfrak{a}_{2}$ is an ideal in a field $\widetilde{F}_{2}$, then we shall write $a_{1}=a_{2}$ provided $a_{1}$ and $a_{2}$ generate the same ideal in some field containing all the numbers of $\widetilde{F}_{1}$ and of $\mathfrak{F}_{2}$ (see [1, §37]). Two such ideals may therefore be denoted by the same symbol and we shall speak of an ideal a without regard to a particular field. An ideal $a$ is said to be contained in a field $\widetilde{F}$ if it may be generated by numbers in $\mathfrak{F}$, that is to say, if it has a basis in $\mathfrak{F}$.

Let $\mathfrak{a}$ be an ideal contained in the fields $\widetilde{\mho}_{1}$ and $\widetilde{F}_{2}$. We say that $\widetilde{F}_{1}$ and $\mathfrak{F}_{2}$, have corresponding residue systems modulo a if for every integer $\alpha_{1}$ of $\mathfrak{\mho}_{1}$ there exists an integer $\alpha_{2}$ of $\mathfrak{\mho}_{2}$ such that $\alpha_{1} \equiv \alpha_{2}(\bmod$ a), and for every integer $\alpha_{2}$ of $\widetilde{F}_{2}$ there exists an integer $\alpha_{1}$ of $\widetilde{F}_{1}$ such that $\alpha_{1} \equiv \alpha_{2}(\bmod a)$.

The problem considered in this paper is the following one: if $\Im_{1}$ and $\widetilde{F}_{2}$ are two fields containing an ideal $\mathfrak{a}$, under what conditions will $\widetilde{F}_{1}$ and $\widetilde{F}_{2}$ have corresponding residue systems mod a. We shall show that this problem reduces to that in which the ideal $a$ is a power of a prime ideal and a necessary and sufficient condition for $\widetilde{F}_{1}$ and $\widetilde{F}_{2}$ to have corresponding residue systems mod $a$ is derived in case that $a$ is a prime ideal. A necessary (but not sufficient) condition is derived in case $\mathfrak{a}$ is a power of a prime ideal and $\widetilde{F}_{1}$ and $\widetilde{F}_{2}$ are normal over $\widetilde{F}_{1} \cap$ $\mathfrak{F}_{2}$. A special case in which the fields are of the type $\mathfrak{F}(\sqrt[q]{\mu} \mu)$ is considered. These fields are of interest in themselves and in view of Corollary 7.1 seem to have a direct connection with the general problem.

THEorem 1. Let a be an ideal in the number fields $\widetilde{F}_{1}$ and $\mathfrak{F}_{2}$ and suppose $\widetilde{F}_{1}$ and $\widetilde{F}_{2}$ have corresponding residue systems mod $\mathrm{\pi}$. Then a has the same prime ideal decomposition in $\widetilde{F}_{1}$ and in $\widetilde{F}_{2}$.

Received May 28, 1954. 
Proof. Let

$$
\begin{aligned}
& \mathfrak{a}=\mathfrak{p}_{1}^{e_{1}} \cdot \ldots \cdot \mathfrak{p}_{r}^{e_{r}} \text { in } \mathfrak{F}_{1} \\
& \mathfrak{a}=\mathfrak{q}_{1}^{f_{1}} \cdot \ldots \cdot \mathfrak{q}_{s}^{f_{s}} \text { in } \mathfrak{F}_{2}
\end{aligned}
$$

where the $\mathfrak{p}_{i}$ are prime ideals in $\mathfrak{F}_{1}$ and the $\mathfrak{q}_{i}$ are prime ideals in $\mathfrak{F}_{2}$. Let $\alpha$ be an integer in $\mathfrak{F}_{1}$ such that $\alpha$ is exactly divisible by $\mathfrak{p}_{1}$ and $\left(\alpha, \mathfrak{p}_{i}\right)=(1)$ for $\mathrm{i}=2, \cdots, r$. There exists an integer $\beta$ in $\mathfrak{F}_{2}$ such that $\alpha \equiv \beta(\bmod a)$ and thus in $\mathfrak{F}_{1} \cup \mathfrak{F}_{2}$ we have $(\beta, \mathfrak{a})=\mathfrak{p}_{1}$. Since $\beta$ is in $\mathfrak{F}_{2}$ and $a \subset \mathfrak{F}_{2}$, it follows that $\mathfrak{p}_{1} \subset \mathfrak{F}_{2}$. In the same manner it follows that $\mathfrak{p}_{i} \subset \mathfrak{F}_{2}$ for $i=1, \cdots, r$ and $\mathfrak{q}_{i} \subset \mathfrak{F}_{1}$ for $i=1, \cdots, s$. Therefore in $\mathfrak{F}_{1}$ and in $\mathfrak{F}_{2}$ we have $\mathfrak{p}_{1}^{e_{1}} \cdot \ldots \cdot \mathfrak{p}_{r}^{e}=\mathfrak{q}_{1}^{f_{1}{ }^{3}} \cdot \ldots \cdot \mathfrak{q}_{s}^{f_{s}}$.

In $\mathfrak{F}_{2}$ the $\mathfrak{q}_{i}$ are prime ideals and hence $\mathfrak{q}_{1} \mid \mathfrak{p}_{j}$ in $\mathfrak{F}_{2}$ for some $j$. In $\widetilde{\mho}_{1}$ the $\mathfrak{p}_{i}$ are prime ideals and therefore $\mathfrak{p}_{k} \mid \mathfrak{q}_{1}$ in $\mathfrak{F}_{1}$ for some $k$. Thus in $\widetilde{\mho}_{1} \cup \mathfrak{F}_{2}$ we have $\mathfrak{p}_{k} \mid \mathfrak{p}_{j}$ which implies that $\mathfrak{p}_{k}=\mathfrak{p}_{j}=\mathfrak{q}_{1}$ in $\mathfrak{F}_{1}$ and in $\mathfrak{F}_{2}$. By renumbering and repeated application of the above argument we obtain $r=s$ and $\mathfrak{p}_{i}=\mathfrak{q}_{i}$ for $i=1, \cdots, r=s$ in $\mathfrak{F}_{1}$ and $\mathfrak{F}_{2}$.

TheoRem 2. Let a be an ideal in the number fields $\mathfrak{F}_{1}$ and $\mathfrak{F}_{2}$. In order that $\mathfrak{F}_{1}$ and $\mathfrak{F}_{2}$ have corresponding residue systems mod $\mathfrak{a}$ it is necessary and sufficient that $\mathfrak{a}=\mathfrak{p}_{1}^{e_{1}} \cdot \ldots \cdot \mathfrak{p}_{r}^{e_{r}}$ where $\mathfrak{p}_{i}$ is a prime ideal in $\mathfrak{F}_{1}$ and $\mathfrak{F}_{2}$, and $\mathfrak{F}_{1}$ and $\mathfrak{F}_{2}$ have corresponding residue systems mod $\mathfrak{p}_{i}^{e_{i}}$ for $i=1, \cdots, r$.

Proof. The necessity follows from Theorem 1. Suppose $\mathfrak{a}=\mathfrak{p}_{1}^{e_{1}} \ldots$ - $\mathfrak{p}_{r}^{e}$ in $\mathfrak{F}_{1}$ and in $\mathfrak{F}_{2}$, where $\mathfrak{p}_{i}$ is a prime ideal in $\mathfrak{F}_{1}$ and in $\mathfrak{F}_{2}$, and that $\widetilde{F}_{1}$ and $\widetilde{F}_{2}$ have corresponding residue systems $\bmod \mathfrak{p}_{i}^{e_{i}}$ for $i=1, \cdots$, $r$. Let $\alpha$ be any integer of $\widetilde{\mho}_{1}$. There exist integers $\beta_{i}$ in $\widetilde{F}_{2}$ such that $\alpha \equiv \beta_{i}\left(\bmod \mathfrak{p}_{i}^{e}\right)$ for $i=1, \cdots, r$. By the Chinese remainder theorem there exists an integer $\beta$ in $\mathfrak{F}_{2}$ such that $\beta \equiv \beta_{i}\left(\bmod \mathfrak{p}_{i}^{i}\right)$ for $i=1, \cdots$, $r$ and hence $\alpha \equiv \beta(\bmod \mathfrak{a})$. It follows that $\widetilde{\mho}_{1}$ and $\mathfrak{F}_{2}$ have corresponding residue systems $\bmod a$.

THEOREM 3. Let $\mathfrak{F}_{1}$ and $\mathfrak{F}_{2}$ be two number fields, $\mathfrak{F}=\widetilde{F}_{1} \cap \mathfrak{F}_{2}$, and let $\mathfrak{p}$ be a prime ideal in both $\mathfrak{F}_{1}$ and $\mathfrak{F}_{2}$. Suppose $\mathfrak{F}_{1}$ and $\mathfrak{F}_{2}$ have corresponding residue systems $\bmod \mathfrak{p}^{j}$ and let $\mathfrak{\mho}_{n}$ be the smallest normal extension over $\mathfrak{F}$ containing $\mathfrak{F}_{1}$ and $\mathfrak{F}_{2}$. Then for every automorphism $A$ in the Galois group $\mathbb{S}\left(\mathfrak{F}_{n} \mid \mathfrak{F}\right)$ of $\mathfrak{F}_{n}$ over $\mathfrak{F}$ we have $\alpha_{1}^{A} \equiv \alpha_{1}\left(\bmod \mathfrak{p}^{j}\right)$ and $\alpha_{2}^{A} \equiv \alpha_{2}\left(\bmod \mathfrak{p}^{j}\right)$ for every integer $\alpha_{1}$ in $\widetilde{\mho}_{1}$ and $\alpha_{2}$ in $\mathfrak{F}_{2}$.

Proof. Let $\mathbb{S}_{1}$ and $\mathbb{S}_{2}$ be the subgroups of $\mathfrak{S}_{(}\left(\mathfrak{F}_{n} \mid \mathfrak{F}_{)}\right)$which leave $\mathfrak{F}_{1}$ and $\mathfrak{F}_{2}$ fixed respectively. Since $\mathfrak{F}=\mathfrak{F}_{1} \cap \mathfrak{F}_{2}$ we have by Galois theory that $\mathbb{S}_{1} \cup \mathbb{S}_{2}$, corresponds to $\mathfrak{F}$ under the Galois correspondence between subgroups and subfields. Hence $\mathbb{S}_{1} \cup \mathbb{S}_{2}=\left(S_{S}\left(\mho_{n} \mid \mathfrak{F}\right)\right.$. 
Denote by $\mathfrak{S}_{i}(i=1,2)$ the set of automorphisms $A$ in $\mathfrak{S}\left(\mathfrak{F}_{n} \mid \mathfrak{F}\right)$ such that $\alpha_{i}^{A} \equiv \alpha_{i}\left(\bmod \mathfrak{p}^{j}\right)$ for all integers $\alpha_{i}$ in $\mathfrak{F}_{i}$ for $i=1,2$. The sets $\mathfrak{S}_{i}$ are subgroups of $\left.\mathfrak{S}_{(\mathfrak{F}}\left|\mathfrak{F}_{n}\right| \mathfrak{F}\right)$. Furthermore the sets $\mathfrak{S}_{i}$ contain $\mathfrak{S}_{i}$ for $i=1,2$.

Let $A$ be an automorphism of $\mathfrak{S}_{2}$. For every integer $\alpha_{1}$ in $\mathfrak{F}_{1}$ there exists an integer $\alpha_{2}$ in $\mathfrak{F}_{2}$ such that $\alpha_{1} \equiv \alpha_{2}\left(\bmod \mathfrak{p}^{j}\right)$. Therefore $\left(\alpha_{1}-\right.$ $\left.\alpha_{2}\right)^{A} \equiv 0\left(\bmod \mathfrak{p}^{j}\right), \alpha_{1}^{A} \equiv \equiv \alpha_{2}^{A}\left(\bmod \mathfrak{p}^{j}\right), \quad \alpha_{1}^{A} \equiv \alpha_{2}\left(\bmod \mathfrak{p}^{j}\right)$, and thus $\alpha_{1}^{A} \equiv \alpha_{1}$ $\left(\bmod \mathfrak{p}^{j}\right)$. Hence the automorphism $A$ is also in $\widetilde{S}_{1}$ and it follows that $\mathfrak{S}_{2} \subset \mathfrak{S}_{1}$. Similarly $\mathfrak{S}_{1} \subset \mathfrak{S}_{2}$ and therefore $\mathfrak{S}_{1}=\mathfrak{S}_{2}$. Hence $\mathfrak{S}_{1}=\mathfrak{S}_{2}=\mathbb{S}\left(\mathfrak{F}_{n}\right)$ F) since $\mathfrak{S}_{i} \supset \mathscr{S}_{i}$ for $i=1,2$ and $\mathscr{S}_{1} \cup \mathscr{S}_{2}=\mathscr{S}\left(\mathfrak{F}_{n} \mid \mathfrak{\Im}\right)$.

COROLlary 3.1. Under the conditions of Theorem 3 it follows that $\mathfrak{D}_{1} \equiv 0\left(\bmod \mathfrak{p}^{n_{1} j}\right)$ and $\mathfrak{D}_{2} \equiv 0\left(\bmod \mathfrak{p}^{n_{2} j}\right)$, where $n_{1}+1=\left(\mathfrak{F}_{1} \mid \mathfrak{F}\right), n_{2}+1=\left(\mathfrak{F}_{2} \mid \mathfrak{F}\right)$, and $\mathfrak{D}_{i}$ denotes the relative differente of $\mathfrak{F}_{i}$ over $\mathfrak{F}$ for $i=1,2$.

THEOREM 4. Let $\mathfrak{F}_{1} \supset \mathfrak{F}$ be two number fields and let $\mathfrak{g}$ be a prime ideal in $\mathfrak{F}_{1}$. Suppose that for every integer $\alpha$ in $\mathfrak{F}_{1}$ we have $\alpha \equiv \alpha^{(i)}$ $(\bmod \mathfrak{B})$ for $i=1, \cdots, k=\left(\mathfrak{F}_{1} \mid \mathfrak{F}\right)$, where $\alpha^{(i)}$ is the $i^{\text {th }}$ conjugate of $\alpha$ in $\mathfrak{F}_{1}$ over $\mathfrak{F}$. Then $\mathfrak{P}$ is of order $k=\left(\mathfrak{F}_{1} \mid \mathfrak{F}\right)$ with respect to $\mathfrak{F}$.

Proof. It is clear that $\mathfrak{P}$ coincides with its conjugates. Moreover if $\alpha$ is any integer in $\widetilde{\mho}_{1}$ and $\alpha_{2}, \cdots, \alpha_{k}$ its conjugates over $\mathfrak{F}$ then

$$
f(x)=(x-\alpha)\left(x-\alpha_{2}\right) \cdots\left(x-\alpha_{k}\right) \equiv(x-\alpha)^{k} \quad(\bmod \mathfrak{P}) .
$$

The polynomial $f(x)$ has its coefficients in $\widetilde{F}$ and since the field of residue classes mod $\mathfrak{P}$ is separable over the field or residue classes mod $\mathfrak{p}$, it must be of degree one.

THEOREM 5. Let $\widetilde{F}_{1}$ and $\widetilde{F}_{2}$ be two number fields and $\mathfrak{p}$ a prime ideal in both fields. Then $\mathfrak{F}_{1}$ and $\mathfrak{\mho}_{2}$ have corresponding residue systems $\bmod \mathfrak{p}$ if and only if $\mathfrak{p}$ is of order $\left(\mathfrak{F}_{1} \mid \mathfrak{F}_{1} \cap \mathfrak{F}_{2}\right)$ in $\widetilde{F}_{1}$ over $\mathfrak{F}_{1} \cap \mathfrak{F}_{2}$ and of order $\left(\mathfrak{F}_{2} \mid \mathfrak{F}_{1} \cap \mathfrak{F}_{2}\right)$ in $\mathfrak{F}_{2}$ over $\mathfrak{F}_{1} \cap \mathfrak{F}_{2}$.

Proof. If $\mathfrak{F}_{1}$ and $\mathfrak{F}_{2}$ have corresponding residue systems $\bmod \mathfrak{p}$, it follows immediately from Theorems 3 and 4 that the order of $p$ satisfies the conditions of the theorem.

The converse is clear since $\mathfrak{p}$ is of degree one over $\mathfrak{F}_{1} \cap \mathfrak{\mho}_{2}$ and therefore every residue class mod $\mathfrak{p}$ contains an integer of $\mathfrak{F}_{1} \cap \mathfrak{F}_{2}$.

CoRollary 5.1. Let a be an ideal in the number fields $\widetilde{F}_{1}$ and $\mathfrak{F}_{2}$. If $\mathfrak{F}_{1}$ and $\mathfrak{F}_{2}$ have corresponding residue systems $\bmod a$, then $\left(\mathfrak{F}_{1} \mid \mathfrak{F}_{1} \cap \mathfrak{F}_{2}\right)$ $=\left(\mathfrak{\mho}_{2} \mid \mathfrak{\Im}_{1} \cap \mathfrak{\mho}_{2}\right)$. 
THEOREM 6. Let $\mathfrak{F}_{1}$ and $\mathfrak{F}_{2}$ be two number fields each normal over $\mathfrak{F}=\widetilde{F}_{1} \cap \mathfrak{F}_{2}$ and let $\mathfrak{p}$ be a prime ideal in $\mathfrak{F}_{1}$ and in $\mathfrak{F}_{2}$. In order that $\widetilde{F}_{1}$ and $\mathfrak{F}_{2}$ have corresponding residue systems mod $\mathfrak{p}$ it is necessary and sufficient that the inertial group of $\mathfrak{p}$ in $\mathfrak{F}_{j}$ over $\mathfrak{F}$ be equal to the Galois group of $\mathfrak{F}_{j}$ over $\mathfrak{F}$ for $j=1,2$.

Proof. The condition is sufficient since $\mathfrak{p}$ is of degree one in $\widetilde{F}_{j}$ over $\mathfrak{F}$ if the inertial group of $\mathfrak{p}$ in $\mathfrak{F}_{j}$ over $\mathfrak{F}$ is equal to the Galois group of $\mathfrak{F}_{j}$ over $\mathfrak{F}$ for $j=1,2$.

Suppose $\mathfrak{F}_{1}$ and $\mathfrak{F}_{2}$ have corresponding residue systems mod $\mathfrak{p}$ and let $\widetilde{F}_{i}$ denote the inertial field of $\mathfrak{p}$ in $\widetilde{F}_{1}$ over $\mathfrak{F}$. The order of $\mathfrak{p}$ in $\widetilde{F}_{1}$ over $\widetilde{F}$ is equal to $\left(\widetilde{F}_{1} \mid \widetilde{F}_{i}\right)$ and hence by Theorem 5 we have $\left(\widetilde{F}_{1} \mid \widetilde{F}_{i}\right)$

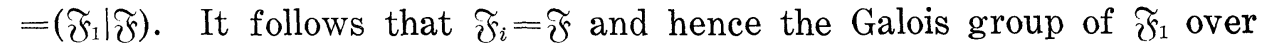
$\mathfrak{F}$ is equal to the inertial group of $\mathfrak{p}$ in $\widetilde{F}_{1}$ over $\widetilde{F}$.

THEOREM 7. Let $\widetilde{F}_{1}$ and $\mathfrak{F}_{2}$ be two number fields each normal over $\mathfrak{F}=\mathfrak{F}_{1} \cap \mathfrak{F}_{2}$, and let $\mathfrak{w}$ be a prime ideal in $\mathfrak{F}_{1}$ and in $\mathfrak{F}_{2}$. If $\mathfrak{F}_{1}$ and $\mathfrak{F}_{2}$ have corresponding resiaue systems mod $\mathfrak{p}^{j}$, then the $j^{\text {th }}$ ramification group of $\mathfrak{p}$ in $\mathfrak{F}_{k}$ over $\mathfrak{F}$ is equal to the Galois group of $\mathfrak{F}_{k}$ over $\mathfrak{F}$ for $k=1,2$.

Proof. Let $A$ be any automorphism of $\left(\mathfrak{S}\left(\mathfrak{F}_{1} \cup \mathfrak{F}_{2} \mid \mathfrak{F}\right)\right.$. It follows from Theorem 3 that $\alpha_{i}^{A} \equiv \alpha_{i}\left(\bmod \mathfrak{p}^{j}\right)$ for every integer $\alpha_{i}$ in $\mathfrak{F}_{i}$ for $i=1,2$. Hence if $A_{i}$ is an automorphism of $\left(\mathfrak{S}\left(\mathfrak{F}_{i} \mid \mathfrak{F}\right),(i=1,2)\right.$, it follows that $\alpha_{i}^{A} \equiv \alpha_{i}\left(\bmod \mathfrak{p}^{j}\right)$ since every automorphism $A_{i}$ of $\mathfrak{S}\left(\mathfrak{F}_{i} \mid \widetilde{F}\right)$ can be continued to an automorphism of $\mathbb{B}\left(\mathfrak{F}_{1} \cup \mathfrak{F}_{2} \mid \mathfrak{F}_{)}\right)$. Thus the $j^{\text {th }}$ ramification group of $\mathfrak{p}$ in $\mathfrak{F}_{i}$ over $\mathfrak{F}$ is equal to the Galois group of $\mathfrak{F}_{i}$ over $\mathfrak{\&}$ for $i=1,2$.

CoROllaRY 7.1. Let $\mathfrak{F}_{1}$ and $\mathfrak{F}_{2}$ be two number fields normal over $\mathfrak{F}=\mathfrak{F}_{1} \cap \mathfrak{F}_{2}$ and let $\mathfrak{p}$ be a prime ideal in $\widetilde{F}_{1}$ and in $\mathfrak{F}_{2}$. If $\mathfrak{F}_{1}$ and $\mathfrak{F}_{2}$ have corresponding residue systems mod $\mathfrak{i}^{j}$ for $j>1$, then $\left(\mathfrak{F}_{1} \mid \mathfrak{F}\right)=\left(\mathfrak{F}_{2} \mid \mathfrak{F}\right)$ $=p^{r}$ where $p$ is the rational prime belonging to $p$.

Proof. By Theorem 7 we have $\mathfrak{G}\left(\mathfrak{F}_{1} \mid \mathfrak{F}\right)=\left(\mathfrak{S}_{1}=\cdots=\mathfrak{G}_{j}\right.$ where $\mathfrak{G}_{j}$ is the $j^{\text {th }}$ ramification group of $\mathfrak{p}$ in $\widetilde{F}_{1}$ over $\mathfrak{F}$. By Theorem 5 the order $e$ of $\mathfrak{p}$ in $\widetilde{F}_{1}$ over $\mathfrak{F}$ is equal to $\left(\mathfrak{F}_{1} \mid \mathfrak{F}\right)$. But $\mathfrak{S}_{1} / \mathfrak{G}_{2}$ is cyclic of order $e_{0}$ where $e=p^{r} e_{0},\left(e_{0}, p\right)=1, p$ the rational prime belonging to the ideal $\mathfrak{p}$. Therefore $\left(\mathfrak{F}_{1} \mid \mathfrak{F}\right)=e_{0} x^{r}$. Since $\left(\mathfrak{S}_{1}=\mathfrak{G}_{2}\right.$ we have $e_{0}=1$ and $\left(\mathfrak{F}_{1} \mid \mathfrak{F}\right)=p^{r}$. Therefore $\left(\mathfrak{F}_{1} \mid \mathfrak{F}\right)=\left(\mathfrak{F}_{2} \mid \mathfrak{F}\right)=p^{r}$.

CoRollary 7.2. Let $\mathfrak{F}_{1}$ and $\mathfrak{F}_{2}$ be two number fields normal over $\mathfrak{\mho}=\widetilde{\mho}_{1} \cap \mathfrak{\mho}_{2}$ and let $\mathfrak{p}$ be a prime ideal in $\widetilde{\mho}_{1}$ and in $\mathfrak{\mho}_{2}$. Let $v_{i}$ denote 
the order of ramification of $\mathfrak{p}$ in $\mathfrak{F}_{i}$ over $\mathfrak{F}$ for $i=1,2$ and suppose $v_{1} \geqq v_{2} \geqq 2$. If $\widetilde{F}_{1}$ and $\widetilde{F}_{2}$ have corresponding residue systems mod $p^{v_{2}}$, then $\mathfrak{G}\left(\mathfrak{F}_{2} \mid \mathfrak{F}_{)}\right)$is Abelian of type $(p, \cdots, p)$ where $p$ is the rational prime belonging to $\mathfrak{p}$.

Proof. If $\mathfrak{F}_{1}$ and $\mathfrak{F}_{2}$ have corresponding residue systems $\bmod \mathfrak{p}^{v_{2}}$, it follows from Theorem 7 that $\left(\mathfrak{S}\left(\mathfrak{F}_{2} / \mathfrak{F}\right)=\mathfrak{S}_{1}=\cdots=\left(\mathfrak{S}_{v_{2}}\right.\right.$ where $\mathfrak{S S}_{j}$ is the $j^{\text {th }}$ ramification group of $\mathfrak{p}$ in $\widetilde{F}_{2}$ over $\mathfrak{F}$. By the definition of $v_{2}$, $\quad \mathfrak{G}_{v_{2}+1}$ is the group identity. But $\mathscr{S}_{v_{2}} / \mathscr{S}_{v_{2}+1}$ is Abelian of type $(p, \cdots, p)$ where $p$ is the rational prime belonging to $\mathfrak{p}$. It follows that $\mathfrak{G}\left(\mathfrak{F}_{2} \mid \mathfrak{F}\right)$ is Abelian of type $(p, \cdots, p)$.

The condition of Theorem 7 is not sufficient as the following example shows. Denote by $R$ the field of rational numbers and let $\widetilde{\mho}_{1}=R$ $(\sqrt{2}), \widetilde{F}_{2}=R(\sqrt{3}), \mathfrak{p}=(\sqrt{ } 2)$. It is clear that the second ramification group of the ideal $\left(V_{2}\right)$ in $\widetilde{F}_{1}$ over $R$ is equal to the Galois group of $\mathfrak{F}_{1}$ over $R$, and likewise for $\mathfrak{F}_{2}$. However $\widetilde{F}_{1}$ and $\mathfrak{F}_{2}$ do not have corresponding residue systems $\bmod (\sqrt{2})^{2}$.

In the remainder of this paper we consider fields of the type $\mathfrak{F}(\sqrt[q]{\mu})$ where $\mathfrak{F}$ is a number field containing a $q^{t h}$ root of unity $\zeta \neq 1$, $q$ is a rational prime, and $\mu$ is an integer of $\widetilde{F}$ and not the $q^{t h}$ power of an integer in $\mathfrak{F}$.

Let $\mathfrak{F}$ be a prime ideal in $\mathfrak{F}\left(\sqrt[q]{\mu_{1}}\right)$ and in $\mathfrak{F}\left(\sqrt[q]{\mu_{2}}\right)$. We may suppose that $\mathfrak{F}\left(\sqrt[q]{\mu_{1}}\right) \neq \mathfrak{F}\left(\sqrt[q]{\mu_{2}}\right)$ since the problem of corresponding residue systems is trivial in case equality holds. By Theorem 5, in order that $\mathfrak{F}\left(\sqrt[q]{\mu_{1}}\right)$ and $\mathfrak{F}\left(\sqrt[q]{\mu_{2}}\right)$ have corresponding residue systems $\bmod \mathfrak{F}$ it is necessary and sufficient that $\mathfrak{F}$ be of order $q$ in $\mathfrak{F}\left(\sqrt[q]{\mu_{1}}\right)$ over $\mathfrak{F}$ and in $\mathfrak{F}\left(\sqrt[q]{\mu_{2}}\right)$ over $\mathfrak{F}$. Therefore it is necessary and sufficient that $\mathfrak{P}$ divide the relative differente $\mathfrak{D}_{i}$ of $\mathfrak{F}\left(\sqrt[q]{ } \mu_{i}\right)$ over $\mathfrak{F}$ for $i=1,2$. If $c_{i}$ denotes the relative conductor of $\sqrt[q]{\mu_{i}}$ for $i=1,2$ then

$$
\left(\sqrt[q]{\mu_{i}}\right)^{q-1} q=\mathfrak{c}_{i} \grave{D}_{i}
$$

for $i=1,2$ since $\left(\sqrt[q]{\mu_{i}}\right)^{q-1} q$ is the relative number differente of $\sqrt[q]{\mu_{i}}$ over $\mathfrak{F}$. It follows that $\mathfrak{P}$ must divide $\left(\sqrt[q]{\mu_{i}}\right)^{q-1} q$ for $i=1,2$ if $\mathfrak{F}\left(\sqrt[q]{\mu_{1}}\right)$ and $\mathfrak{F}\left(\sqrt[q]{\mu_{2}}\right)$ have corresponding residue systems $\bmod \mathfrak{P}$.

Denote by $\mathfrak{p}$ the prime ideal corresponding to $\mathfrak{F}$ in $\mathfrak{F}$. If $\mathfrak{p}$ divides $\mu_{i}$ but not $q$ then $\mathfrak{p}=\mathfrak{P}^{q}$ in $F\left(\sqrt[q]{\mu_{i}}\right)$ if and only if $\left(\mu_{i}\right)=\mathfrak{p}^{a_{i}} \mathfrak{a}_{i}$ for $i=1,2$ where $\left(a_{i}, q\right)=1$ and $\left(a_{i}, \mathfrak{p}\right)=(1)$. (See $\left.[1, p .150]\right)$. Thus we have the following theorem. 
THEOREM 8. If $(\mathfrak{P}, q)=(1)$, then $\mathfrak{F}\left(\sqrt[q]{\mu_{1}}\right)$ and $\mathfrak{F}\left(\sqrt[q]{\mu_{2}}\right)$ have corresponding residue systems mod $\mathfrak{P}$ if and only if $\left(\mu_{i}\right)=\mathfrak{p}^{a_{i}} \mathfrak{a}_{i}$ with $\left(a_{i}, q\right)$ $=1$ and $\left(\mathfrak{a}_{i}, \mathfrak{p}\right)=(1)$ for $i=1,2$.

From Corollary 7.1 it follows that $\mathfrak{F}\left(\sqrt[q]{\mu_{1}}\right)$ and $\mathfrak{F}\left(\sqrt[q]{\mu_{2}}\right)$ do not have corresponding residue systems $\bmod \mathfrak{P}^{j}$ for $j>1$ in case $(\mathfrak{P}, q)=(1)$.

We now consider prime ideals in fields $\widetilde{F}(q / \mu)$ which divide $q$, that is, prime ideals which divide the ideal $(1-\zeta)$ where $\zeta \neq 1$ is a $q^{\text {th }}$

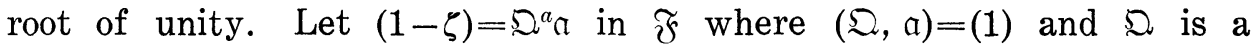
prime ideal in $\mathfrak{F}$, and let $\mathfrak{q}$ be a prime ideal of $F(\sqrt[q]{ } \mu)$ which divides

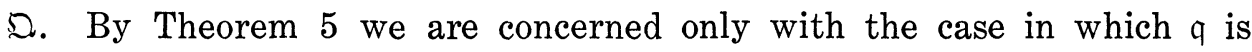
of order $q$ in $\mathfrak{F}(\sqrt[q]{\mu})$ over $\mathfrak{F}$, that is $\mathfrak{Q}=\mathfrak{q}^{q}$ in $\mathfrak{F}(\sqrt[q]{\mu})$. We may suppose without loss of generality that either $(\mu, \mathfrak{Q})=(1)$ or $\left(\mu, \mathfrak{\Omega}^{2}\right)=\mathfrak{Q}$. The ideal $\precsim$ becomes the $q^{\text {th }}$ power of a prime ideal in $\mathfrak{F}(\sqrt[q]{\mu})$ in

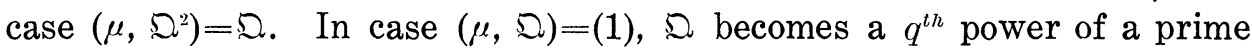
ideal in $\mathfrak{F}(\sqrt[q]{\mu})$ if the congruence $\mu \equiv \xi^{q}\left(\bmod \mathfrak{D}^{a q}\right)$ is not solvable for $\xi$ in $\mathfrak{F}$.

The main result of this paper for fields of the type $\mathfrak{F}(\sqrt[q]{\mu})$ is the following one: if $\mu_{1}, \mu_{2}$ are two integers of $\mathfrak{F}$ such that $\mathfrak{D}=q^{q}$ in $\mathfrak{F}\left(\sqrt[q]{\mu_{1}}\right)$ and in $\mathfrak{F}\left(\sqrt[q]{\mu_{2}}\right)$, and $\mathfrak{q}$ has ramification orders $\geqq v>a$ in $\mathfrak{F}\left(\sqrt[q]{\mu_{1}}\right)$ and in $\mathfrak{F}\left(\sqrt[q]{\mu_{2}}\right)$ over $\mathfrak{F}$ then $\mathfrak{F}\left(\sqrt[q]{\mu_{1}}\right)$ and $\mathfrak{F}\left(\sqrt[q]{\mu_{2}}\right)$ have corresponding residue systems $\bmod \mathfrak{q}^{v-a}$.

We first consider the case in which $\left(\mu, \mathfrak{Q}^{2}\right)=\mathfrak{Q}$

THEOREM 9. If $\left(\mu, \mathfrak{Q}^{2}\right)=\mathfrak{D}$ and $n$ is a positive integer, then $\mathfrak{Q}=\mathfrak{q}^{q}$ in $\mathfrak{F}(\sqrt[q]{\mu})$ and every integer $\alpha$ in $\mathfrak{F}(\sqrt[q]{\mu})$ satisfies a congruence

$$
\alpha \equiv \alpha_{0}+\alpha_{1} \sqrt[q]{\mu}+\cdots+\alpha_{n-1} \sqrt[q]{\mu^{n-1}}\left(\bmod \mathfrak{q}^{n}\right)
$$

where the $\alpha_{i}$ are integers in $\mathfrak{F}$. Furthermore the order of ramification $v$ of $\mathfrak{q}$ in $\mathfrak{F}(\sqrt[q]{\mu})$ over $\mathfrak{F}$ is equal to $a q+1$.

Proof. Since $\left(\mu, \mathfrak{Q}^{2}\right)=\mathfrak{Q}$, we have $\mathfrak{Q}=\mathfrak{q}^{q}$ in $\mathfrak{F}(\sqrt[q]{\mu})$ where $\mathfrak{q}$ is a prime ideal. It follows that $\sqrt[q]{\mu}$ is exactly divisible by $q$. Let $n$ be any positive integer. If $\alpha$ is any integer of $\mathfrak{F}$ we have

$$
\alpha \equiv \alpha_{0}+\alpha_{1} \sqrt[q]{\mu}+\cdots+\alpha_{n-1} \sqrt[q]{\mu^{n-1}}\left(\bmod \mathfrak{q}^{n}\right)
$$

where the $\alpha_{i}$ are residues mod $\mathfrak{q}$ and may be chosen in $\mathfrak{F}$ since $\mathfrak{q}$ is of degree 1 with respect to $\mathfrak{F}$.

The order of ramification of $\mathfrak{q}$ is equal to $v$ if and only if

$$
\sqrt[q]{\mu} \equiv \zeta \sqrt[q]{\mu}\left(\bmod \mathfrak{q}^{v}\right) \text { and } \sqrt[q]{\mu} \neq \zeta \sqrt[q]{\mu}\left(\bmod \mathfrak{q}^{v+1}\right) \text {. }
$$

Hence $v=a q+1$ since $(1-\zeta)=\mathfrak{Q}^{a} \mathfrak{a}, \mathfrak{l}=\mathfrak{q}^{q}$, and $(\mathfrak{a}, \mathfrak{a})=(1)$. 
THEOREM 10. If $\mu_{1}, \mu_{2}$ are two integers of $\mathfrak{\&}$ each exactly divisible by $\mathfrak{\Omega}$, then $\mathfrak{F}\left(\sqrt[q]{V} \mu_{1}\right)$ and $\mathfrak{F}\left(\sqrt[q]{\mu_{2}}\right)$ have corresponding residue systems $\bmod \mathfrak{q}^{a q+1-a}$.

Proof. Choose a fixed residue system $\bmod \mathfrak{d}$ in $\mathfrak{F}$ consisting of $q^{\text {th }}$ powers, which is possible since $\mathfrak{D}$ is a prime ideal in $\mathfrak{F}$. Represent the residue class 0 by 0 and let $n=a(q-1)$. Since $\mu_{1}$ is exactly divisible by $\cong$ we have

$$
\mu_{2} \equiv \alpha_{1}^{q} \mu_{1}+\cdots+\alpha_{n}^{q} \mu_{1}^{n} \quad\left(\bmod \varrho^{n+1}\right)
$$

where the $\alpha_{i}^{q}$ belong to the fixed residue system mod $\mathfrak{D}$ chosen above. Hence

$$
\begin{aligned}
& \left(\sqrt[q]{ } \mu_{2}-\alpha_{1} \sqrt[q]{\mu_{1}}-\cdots-\alpha_{n} \sqrt[q]{\mu_{1}^{n}}\right)^{q} \\
& \equiv \mu_{2}-\alpha_{1}^{q} \mu_{1}-\cdots-\alpha_{n}^{q} \mu_{1}^{n} \quad\left(\bmod \Omega^{n+1}\right) \\
& \equiv 0 \quad\left(\bmod \mathfrak{\Omega}^{n+1}\right) .
\end{aligned}
$$

It follows that

$$
\sqrt[q]{\mu_{2}} \equiv \alpha_{1} \sqrt[q]{\mu_{1}}+\cdots+\alpha_{n} \sqrt[q]{\mu_{1}^{n}}\left(\bmod \mathfrak{q}^{n+1}\right)
$$

and by Theorem 9 , $\mathfrak{F}\left(\sqrt[q]{\mu_{1}}\right)$ and $\mathfrak{F}\left(\sqrt[q]{\mu_{2}}\right)$ have corresponding residue systems $\bmod \mathfrak{q}^{a q+1-a}$.

By Theorem 7 the fields $\mathfrak{F}\left(\sqrt[q]{\mu_{1}}\right)$ and $\mathfrak{F}\left(\sqrt[q]{\mu_{2}}\right)$ do not have corresponding residue systems mod $\mathfrak{q}^{v+1}$ where $v$ is the order of ramification of $\mathfrak{q}$. The following theorem gives a sufficient condition for $\mathfrak{F}\left(\sqrt[q]{\mu_{1}}\right)$ and $\mathfrak{F}\left(\sqrt[q]{q} \mu_{2}\right)$ to have corresponding residue systems $\bmod \mathfrak{q}^{v}$.

THEOREM 11. Let $\mu_{1}, \mu_{2}$ be two integers of $\mathfrak{F}$ each exactly divisible

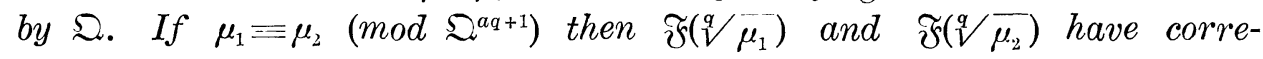
sponding residue systems $\bmod \mathfrak{q}^{a+1}$, that is, mod $\mathfrak{q}^{v}$ where $v$ is the order of ramification of $\mathfrak{q}$.

Proof. Since $\mu_{1} \equiv \mu_{2}\left(\bmod \complement_{\mathfrak{i}^{a q+1}}\right)$ and $\left(\sqrt[q]{\mu_{1}}-\sqrt[q]{\mu_{2}}\right)^{q} \equiv \mu_{1}-\mu_{2}(\bmod q)$

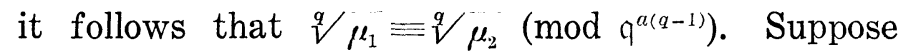

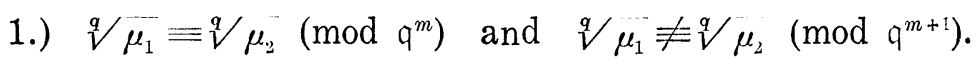

For any polynomial $p(x, y)$ with integral coefficients such that $y$ occurs in every term we have $q p\left(\sqrt[q]{\mu_{1}}, \sqrt[q]{\mu_{2}}\right) \equiv q p\left(\sqrt[q]{\mu_{2}}, \sqrt[q]{\mu_{2}}\right)\left(\bmod \mathfrak{q}^{m+1} q\right)$.

Thus $\quad\left(\sqrt[q]{\mu_{1}}-\sqrt[q]{\mu_{2}}\right)^{q} \equiv \mu_{1}-\mu_{2}\left(\bmod q q^{m} \mathfrak{q}\right)$.

2.) $\left(\sqrt[q]{\mu_{1}}-\sqrt[q]{\mu_{2}}\right)^{q} \equiv \mu_{1}-\mu_{2}\left(\bmod \mathfrak{\Omega}^{a(q-1)} \mathfrak{q}^{m} \mathfrak{q}\right)$.

If $\mu_{1}-\mu_{2} \neq 0\left(\bmod \mathfrak{\Omega}^{a(q-1)} \mathfrak{q}^{m} \mathfrak{q}\right)$ then 


$$
q(a q+1)<a q(q-1)+m+1 \quad \text { since } \quad \mu_{1} \equiv \mu_{2}\left(\bmod \mathfrak{\Omega}^{a q+1}\right) .
$$

Therefore $q<-a q+m+1$ and $m \geqq a q+1$. On the other hand if $\mu_{1}-\mu_{2} \equiv 0\left(\bmod \subseteq^{a(q-1)} \mathfrak{q} \mathfrak{q}\right)$ then

$$
\left(\sqrt[q]{ } \mu_{1}-\sqrt[q]{\mu_{2}}\right)^{q} \equiv \equiv \quad\left(\bmod \mathfrak{\complement}^{a(q-1)} \mathfrak{q}^{m} \mathfrak{q}\right)
$$

from 2.). Thus by 1.) we have $m q \geqq a q(q-1)+m+1, m>a q$, and hence $m \geq a q+1$. Therefore in either case $m \geqq a q+1$ and we have by 1 .)

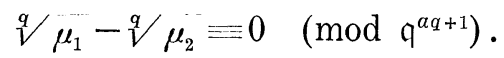

Let $\alpha$ be any integer of $\mathfrak{F}\left(\sqrt[q]{\mu_{1}}\right)$ and $v$ the order of ramification of $\mathfrak{q}$, that is, $v=a q+1$. By Theorem 9

$$
\alpha \equiv \alpha_{0}+\alpha_{1} v^{q} \mu_{1}+\cdots+\alpha_{v-1} \frac{q}{\mu_{1}^{v-\overline{1}}}\left(\bmod \mathfrak{q}^{v}\right)
$$

where the $\alpha_{i}$ are integers in $\mathfrak{F}$. Let

$$
\beta=\alpha_{0}+\alpha_{1} \sqrt[q]{\mu_{2}}+\cdots+\alpha_{v-1} \sqrt[q]{\mu_{2}^{v-1}} .
$$

Then $\alpha \equiv \beta\left(\bmod \mathfrak{q}^{v}\right)$ and $\mathfrak{F}\left(\sqrt[q]{\mu_{1}}\right)$ and $\mathfrak{F}\left(\sqrt[q]{\mu_{2}}\right)$ have corresponding residue systems $\bmod \mathfrak{q}^{v}$.

The condition $\mu_{1} \equiv \mu_{2}\left(\bmod \Sigma^{a q+1}\right)$ in Theorem 11 may be replaced by $\mu_{1} \equiv \mu_{2} \sigma^{q}\left(\bmod \mathfrak{\Omega}^{a q+1}\right)$ where $\sigma$ is in $\widetilde{F}$.

We now consider the case in which $(\mu, \mathfrak{Q})=(1)$ and the congruence $\mu \equiv \xi^{q}\left(\bmod \mathfrak{\Omega}^{a q}\right)$ is not solvable for $\xi$ in $\mathfrak{F}$, that is, $(\mu . \mathfrak{D})=(1)$ and $\mathfrak{Z}=\mathfrak{q}^{\alpha}$ in $\mathfrak{F}(\sqrt[q]{ } \mu)$. Let $k$ be the largest integer such that the congruence $\mu \equiv \xi^{q}\left(\bmod \mathfrak{i}^{k}\right)$ is solvable for $\xi$ in $\mathfrak{F}$. Clearly $0<k<a q$ and $k$ is the largest integer such that the congruence $q \mu \equiv \xi\left(\bmod q^{k}\right)$ is solvable for $\xi$ in $\mathfrak{F}$.

THEOREM 12. Let $\mu$ be an integer of $\mathfrak{F}$ such that $(\mu, \mathfrak{Q})=(1)$ and $\mathfrak{Q}=\mathfrak{q}^{q}$ in $\mathfrak{F}(\sqrt[q]{\mu})$. Let $k$ be the largest integer such that $\mu \equiv \xi^{q}(\bmod$ $\left.\mathfrak{S}^{k}\right)$ is solvable for $\xi$ in $\mathfrak{F}$. Then the order of ramification $v$ of $\mathfrak{q}$ with respect to $\mathfrak{F}$ is equal to $a q+1-k$.

Proof. Let $\alpha$ in $\widetilde{F}$ be a solution of the congruence $\mu \equiv \xi^{q}\left(\bmod \mathfrak{\Omega}^{k}\right)$ with $k$ maximal. Since $\mu-\alpha^{q}$ is exactly divisible by $\Omega^{k}$, it follows that $q \mu-\alpha$ is exactly divisible by $q^{k}$. Furthermore we have $(k, q)=1$ (see [1, p. 153]). Thus there exist positive integers $x$ and $y$ such that $k x=1+q y$.

Let $\pi$ be an integer of $\widetilde{F}$ such that $(\pi)=a \mathfrak{l}$ where $(a, \mathfrak{\Omega})=(1)$ and $a$ is an ideal of $\mathfrak{F}$. There exists an ideal $c$ in $\mathfrak{F}$ such that $a c=(\omega)$ is principal and $c$ is prime to $\Omega$. 
Now, let

$$
\rho=\frac{\left(\sqrt[q]{\mu-\alpha)^{x}}\right.}{\pi^{y}}
$$

Then

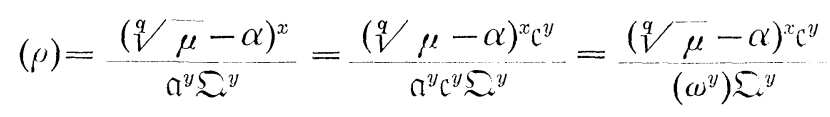

and

$$
\left(\omega^{y} p\right)=\frac{(\sqrt[q]{\mu}-\alpha)^{x} c^{y}}{\Sigma^{y}} .
$$

The ideal fraction on the right in the last equation is an integral ideal exactly divisible by $q$, and therefore $\omega^{y} \rho$ is an integer of $\mathfrak{\&}$ exactly divisible by $q$. It follows that the order of ramification of $\mathfrak{q}$ is equal to $v$ if and only if $\omega^{y} \rho-\left(\omega^{y} \rho\right)^{A}$ is exactly divisible by $q^{v}$ where $A$ is the automorphism $q u \rightarrow \zeta q \mu$, that is, if and only if

$$
\frac{\omega^{y}(\underline{q} \mu-\alpha)^{x}}{\pi^{y}}-\frac{\omega^{y}(\zeta \sqrt[q]{\mu-\alpha})^{x}}{\pi^{y}}
$$

is exactly divisible by $\mathfrak{q}^{v}$. Since $(\omega, \Omega)=(1)$ this is true if and only if $(\sqrt[q]{\mu}-\alpha)^{x}-(\zeta \sqrt[q]{\mu}-\alpha)^{x}$ is exactly divisible by $\coprod^{y} \mathfrak{q}^{v}=\mathfrak{q}^{k x-1} \mathfrak{q}^{v}$. Now

$$
\begin{aligned}
(\zeta \sqrt[q]{\mu}-\alpha)^{x} & =[(\zeta \sqrt[q]{\mu}-\sqrt[q]{\mu})+(\sqrt[q]{\mu}-\alpha)]^{x} \\
& =(\sqrt[q]{\mu-\alpha})^{x}+x(\sqrt[q]{ } \mu-\alpha)^{x-1}(\zeta \sqrt[q]{\mu-\sqrt[q]{\mu})+\cdots}
\end{aligned}
$$

Therefore

$$
\begin{aligned}
(\zeta q \bar{q}-\alpha)^{x} & \equiv(\sqrt[q]{\mu-\alpha})^{x} \quad\left(\bmod \mathfrak{q}^{k(x-1)}(1-\zeta)\right) \\
& \equiv\left(\sqrt[q]{\mu-\alpha)^{x}} \quad\left(\bmod \mathfrak{q}^{k(x-1)} \mathfrak{q}^{a l}\right)\right.
\end{aligned}
$$

since $0<k<a q$ and $(1-\zeta)=\Omega^{a} a$ with $(\Omega, a)=(1)$. Furthermore this congruence holds exactly $\bmod \mathrm{q}^{k(x-1)} \mathfrak{q}^{a q}$. It follows that $k x-1+v=k(x$ $-1)+a q$ and $v=a q+1-k$.

THEOREM 13. Let $\mu_{1}, \mu_{2}$ be two integers of $\mathfrak{F}$ each prime to $\mathfrak{D}$ and such that $\Omega=q^{q}$ in, $\mathfrak{F}\left(\sqrt[q]{\mu_{1}}\right)$ (and $\mathfrak{F}\left(\sqrt[q]{\mu_{2}}\right)$. Let $k_{i}$ be the largest integer. such that the congruence $\mu_{i} \equiv \alpha_{i}^{q}\left(\bmod \Omega^{k_{i}}\right)$ is solvable for $\alpha_{i}$, an integer of $\mathfrak{F}(i=1,2)$. Let $v_{i}=a q+1-k_{i}$ for $i=1,2$, and suppose $v_{1} \geqq v_{2}>a$. Then $\mathfrak{F}\left(\frac{q}{\mu_{1}}\right)$ and $\mathfrak{F}\left(q^{q} \mu_{2}\right)$ have corresponding residue systems mod $\mathfrak{q}^{v_{2}-a}$. 
Proof. Since $\mu_{i}-\alpha_{i}^{q}$ is exactly divisible by $\bigcap^{k_{i}}$ it follows that $\sqrt[q]{\mu_{i}}-\alpha_{i}$ is exactly divisible by $q^{k_{i}}$ for $i=1,2$. Since $\left(k_{i}, q\right)=1$ we have positive integers $x_{i}$ and $y_{i}$ such that $k_{i} x_{i}=1+q y_{i}$ for $i=1,2$. Let

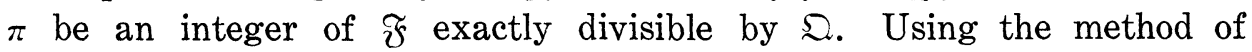
Theorem 12 we obtain an integer

$$
\theta_{i}=\frac{\omega^{y_{i}}\left(\sqrt[q]{\mu_{i}}-\alpha_{i}\right)^{x_{i}}}{\pi^{y_{i}}}
$$

of $\mathfrak{F}\left(\sqrt[q]{\mu_{i}}\right)$ which is exactly divisible by $\mathfrak{q}$ for $i=1,2$.

We now show that $\theta_{i}^{q}$ is congruent to an integer of $\mathfrak{F} \bmod \mathfrak{S}_{i}^{v_{i}^{-a}}$ for $i=1,2$. We have

$$
\theta_{i}^{q}=-\frac{\omega^{y_{i} q}\left(\sqrt[q]{\mu_{i}}-\alpha_{i}\right)^{x_{i} q}}{\pi^{y_{i} q}}=\frac{\omega^{y_{i} q}\left(\lambda_{i}-\rho_{i} q\right)^{x_{i}}}{\pi^{y_{i} q}}
$$

where $\lambda_{i}$ is an integer of $\mathfrak{F}$ and $\lambda_{i} \equiv 0\left(\bmod \mathfrak{\Omega}^{k_{i}}\right)$. Hence since $\rho_{i}$ is divisible by $\mathfrak{q}^{k_{i}}$

$$
\begin{aligned}
\theta_{i}^{q} & =\frac{\omega^{y_{i} q}\left(\lambda_{i}^{x_{i}}-x_{i} \lambda_{i}^{x_{i}-1} \rho_{i} q+\cdots\right)}{\pi^{y_{i}^{q}}} \\
& =\frac{\omega^{y_{i} q} \lambda_{i}^{x_{i}}}{\pi^{y_{i} q}}-\frac{\left(\omega^{y_{i} a} x_{i} \lambda_{i}^{x_{i}-1} \rho_{i} q+\cdots\right)}{\pi^{y_{i} q}} \\
& \equiv \frac{\omega^{y_{i} q} \lambda_{i}^{x_{i}}}{\pi^{y_{i} q}} \quad\left(\bmod \mathfrak{Q}^{a q+1-k_{i}-a}\right) \\
& \equiv \frac{\omega^{y_{i} q} \lambda_{i}^{x_{i}}}{\pi^{y_{i} q}} \quad\left(\bmod \mathfrak{D}^{v_{i}-a}\right)
\end{aligned}
$$

But the expression on the right of the last congruence is an integer of $\mathfrak{F}$, so that $\theta_{i}^{a}$ is congruent to an integer of $\mathfrak{F} \bmod \mathfrak{\Omega}^{v_{i}-a}$.

We now show that the $q^{t h}$ power of every integer of $\mathfrak{F}\left(\sqrt[q]{\mu_{i}}\right)$ is congruent to an integer of $\widetilde{₹} \bmod \mathfrak{Q}^{v_{i}-a}$ for $i=1,2$.

Let $\beta$ be any integer of $\mathfrak{F}\left(\sqrt[q]{\mu_{1}}\right)$ and let $n=v_{1}-a$. Since $\theta_{1}$ is exactly divisible by $\mathfrak{q}$ we have $\beta \equiv \beta_{0}+\beta_{1} \theta_{1}+\cdots+\beta_{n-1} \theta_{1}^{n-1}\left(\bmod \mathfrak{q}^{n}\right)$, where the $\beta_{i}$ are residues mod $\mathfrak{q}$ and may be chosen in $\mathfrak{F}$ since $\mathfrak{q}$ is of degree 1 over $\mathfrak{F}$. Hence

$$
\begin{aligned}
& {\left[\beta-\left(\beta_{0}+\cdots+\beta_{n-1} \theta_{1}^{n-1}\right)\right]^{q} } \\
\equiv & \beta^{q}-\left(\beta_{0}+\cdots+\beta_{n-1} \theta_{1}^{n-1}\right)^{q} \quad(\bmod q) \\
\equiv & \beta^{q}-\left(\beta_{0}^{q}+\cdots+\beta_{n-1}^{q} \theta_{1}^{q(n-1)}\right) \quad(\bmod q) \\
\equiv & \beta^{q}-\sigma \quad \bmod \left(\mathfrak{Q}^{v_{1}-a}\right),
\end{aligned}
$$

where $\sigma$ is an integer of $\mathfrak{F}$. It follows that $\beta^{q} \equiv \sigma\left(\bmod \mathfrak{2}^{v_{1}-a}\right)$. 
If $\beta$ and $\beta^{\prime}$ are two integers of $\mathfrak{F}\left(\frac{q}{\mu_{1}}\right)$ such that $\beta^{q} \equiv \sigma(\bmod$ $\left.\mathfrak{D}^{v_{1}-a}\right)$ and $\beta^{\prime}=\sigma\left(\bmod \mathfrak{l}^{v_{1}-a}\right)$, then $\beta \equiv \beta^{\prime}\left(\bmod q^{v_{1}-a}\right)$. Also if $\beta^{q} \equiv \sigma$ $\left(\bmod \mathfrak{Q}^{v_{1}-a}\right)$ and $\beta^{a} \equiv \sigma^{\prime}\left(\bmod \mathfrak{V}^{v_{1}-a}\right)$ where $\sigma, \sigma^{\prime}$ are integers of $\widetilde{F}$, then $\sigma \equiv \sigma^{\prime}\left(\bmod \mathfrak{l}^{v_{1}-a}\right)$. The number of residue classes $\bmod \mathfrak{q}^{v_{1}-a}$ in $\mathfrak{F}\left(\sqrt[q]{\mu_{1}}\right)$ is equal to the number of residue classes $\bmod \mathfrak{D}^{v_{1}-a}$ in $\mathfrak{F}$. It follows that if $\sigma$ is any integer of $\mathfrak{F}$ there exists an integer $\beta$ of $\mathfrak{F}\left(\sqrt[q]{ } \mu_{1}\right)$ such that ${ }^{a} \beta \equiv \sigma\left(\bmod \mathfrak{V}^{v_{1}-a}\right)$.

Similarly, if $\gamma$ is any integer of $\mathfrak{F}\left(\sqrt[q]{\mu_{2}}\right)$ there exists an integer $\tau$ of $\mathfrak{F}$ such that $\gamma^{q} \equiv \tau\left(\bmod \mathfrak{Q}_{2^{-a}}\right)$. There exists an integer $\beta$ of $\mathfrak{F}\left(\sqrt[q]{\mu_{1}}\right)$ such that $\beta^{q} \equiv \tau\left(\bmod q^{v_{1}-a}\right)$. Since $v_{1} \geqq v_{2}$ we have $\beta^{q} \equiv \gamma^{q}$ $\left(\bmod \mathfrak{Q}^{v_{2}-a}\right)$ and therefore $\beta \equiv \gamma\left(\bmod \mathfrak{q}^{v_{2}-a}\right)$.

THEOREM 14. If $\mu_{1}, \mu_{2}$ are two integers of $\mathfrak{F}$ such that $\mathfrak{Q}=q^{q}$ in $\mathfrak{F}\left(\sqrt[q]{\mu_{1}}\right)$ and in $\mathfrak{F}\left(\sqrt[q]{\mu_{2}}\right)$, and $\mathfrak{q}$ has ramification orders $\geq v>a$ in $\mathfrak{F}\left(\sqrt[q]{\mu_{1}}\right)$, $\mathfrak{F}\left(\sqrt[q]{\mu_{2}}\right)$ over $\mathfrak{F}$, then $\mathfrak{F}\left(\sqrt[q]{\mu_{1}}\right)$ and $\mathfrak{F}\left(\sqrt[q]{\mu_{2}}\right)$ have corresponding residue systems mod $\mathfrak{q}^{v-a}$.

Proof. We need only to consider the case in which $\mu_{1}$ is exactly divisible by $\mathfrak{Q}$ and $\mu_{2}$ is prime to $\mathfrak{Z}$, the other two cases following from Theorems 10 and 13.

Let $v_{1}=a q+1$ be the order of ramification of $\mathfrak{q}$ in $\mathfrak{F}\left(\sqrt[q]{\mu_{1}}\right)$ over $\mathfrak{F}$, and let $v_{2}$ be the order of ramification of $\mathfrak{q}$ in $F\left(\sqrt[q]{V} \mu_{2}\right)$ over $\mathfrak{F}$. From Theorem 12 it follows that $v_{1}-1=a q \geqq v_{2}$.

Let $\alpha$ be any integer of $\mathfrak{F}\left(\sqrt[q]{\mu_{1}}\right)$ and let $n=a q-a$. Since $\sqrt[q]{\mu_{1}}$ is exactly divisible by $\mathfrak{q}$, it follows that

$$
\alpha \equiv \alpha_{0}+\alpha_{1} \sqrt[q]{\mu_{1}}+\cdots+\alpha_{n-1} \sqrt[q]{\mu_{1}^{n-1}}\left(\bmod \mathfrak{q}^{n}\right),
$$

where the $\alpha_{i}$ are integers in $\mathfrak{F}$. Hence

$$
\begin{aligned}
\alpha^{q} & \equiv \alpha_{0}^{q}+\alpha_{1}^{q} \mu_{1}+\cdots+\alpha_{n-1}^{q} \mu_{1}^{n-1} \quad\left(\bmod \mathfrak{\Omega}^{n}\right) \\
& \equiv \sigma \quad\left(\bmod \mathfrak{\Omega}^{a q-a}\right)
\end{aligned}
$$

where $\sigma$ is an integer of $\mathfrak{F}$. Using the method of Theorem 13, there exists an integer $\beta$ of $\Im\left(\sqrt[q]{\mu_{2}}\right)$ such that $\beta^{q} \equiv \sigma\left(\bmod \mathfrak{Q}^{v_{2}-a}\right)$. Therefore $\alpha^{q} \equiv \beta^{q}\left(\bmod \mathfrak{D}^{v_{2}-a}\right)$ and $\alpha \equiv \beta\left(\bmod \mathfrak{q}^{v_{2}-a}\right)$. Thus $\mathfrak{F}\left(\sqrt[q]{\mu_{1}}\right)$ and $\mathfrak{F}\left(\sqrt[q]{\mu_{2}}\right)$ have corresponding residue systems mod $q^{v-a}$ where $v_{2} \geqq v>a$.

TheOREM 15. Let $\mu_{1}, \mu_{2}$ be two integers of $\mathfrak{F}$, each prime to $\Omega$, such that $\mathfrak{Q}=\mathfrak{q}^{q}$ in $\mathfrak{F}\left(\sqrt[q]{\mu_{1}}\right)$ and in $\mathfrak{F}\left(\sqrt[q]{\mu_{2}}\right)$. Suppose $\mu_{1} \equiv \mu_{2}(\bmod$ $\left.\Omega^{a q}\right)$ and let $k$ be the largest integer such that the congruences $\mu_{1} \equiv \alpha^{q}$ $\left(\bmod \Omega^{k}\right)$ and $\mu_{2} \equiv \alpha^{q}\left(\bmod \Omega^{k}\right)$ are solvable for $\alpha$ an integer of $\mathfrak{F}$. 
Then $\mathfrak{F}\left(\frac{q}{\mu_{1}}\right)$ and $\mathfrak{F}\left(\sqrt[q]{\mu_{2}}\right)$ have corresponding residue systems $\bmod q^{v}$ where $v=\alpha q+1-k$.

Proof. Since $\mu_{1} \equiv \mu_{2}\left(\bmod \Im^{a q}\right)$ it follows that $\sqrt[q]{\mu_{1}} \equiv \sqrt[q]{\mu_{2}}(\bmod$ $\left.\mathfrak{\Omega}^{a}\right)$ using the method of Theorem 11. We have $k x=1+q y$ and following Theorem 12 it is sufficient to show that

$$
\left(\sqrt [ q ] { \mu _ { 1 } - \alpha ) ^ { x } } \equiv \left(\sqrt[q]{\left.\mu_{2}-\alpha\right)^{x}} \quad\left(\bmod q^{v+q y}\right)\right.\right.
$$

We have

$$
\begin{aligned}
\left(\sqrt[q]{\mu_{2}}-\alpha\right)^{x} & =\left[\left(\sqrt[q]{\mu_{1}}-\alpha\right)+\left(\sqrt[q]{\mu_{2}}-\sqrt[q]{\mu_{1}}\right)\right]^{x} \\
& =\left(\sqrt[q]{\mu_{1}}-\alpha\right)^{x}+x\left(\sqrt[q]{\mu_{1}}-\alpha\right)^{x-1}\left(\sqrt[q]{\mu_{2}}-\sqrt[q]{\mu_{1}}\right)+\cdots \\
& =\left(\sqrt[q]{\mu_{1}}-\alpha\right)^{x} \quad\left(\bmod \mathfrak{q}^{k(x-1)} \mathfrak{q}^{a q}\right) \\
& \equiv\left(\sqrt[q]{\mu_{1}}-\alpha\right)^{x} \quad\left(\bmod \mathfrak{q}^{v+q y}\right)
\end{aligned}
$$

Thus $\mathfrak{F}\left(\sqrt[q]{\mu_{1}}\right)$ and $\mathfrak{F}\left(\sqrt[q]{\mu_{2}}\right)$ have corresponding residue systems $\bmod \mathfrak{q}^{v}$ where $v=a q+1-k$ is the order of ramification of $q$ in $\mathfrak{F}\left(\sqrt[q]{\mu_{1}}\right)$ and $\mathfrak{F}\left(\sqrt[q]{\mu_{2}}\right)$ over $\mathfrak{F}$.

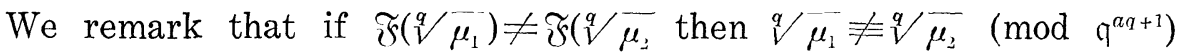
for otherwise we would have corresponding residue systems mod $q^{v+1}$ contrary to Theorem 7 .

In Theorem 15 we may replace the condition $\mu_{1} \equiv \mu_{2}\left(\bmod \Im^{a q}\right)$ by $\mu_{1} \equiv \mu_{2} \beta^{q}\left(\bmod \mathfrak{Q}^{a q}\right)$ with $\beta$ in $\mathfrak{F}$.

THEOREM 16. Let $\mu_{1}, \mu_{2}$ be two integers of $\widetilde{\&}$ such that $\mathfrak{Q}=q^{q}$ in $\mathfrak{F}\left(\sqrt[q]{\mu_{1}}\right)$ and in $\mathfrak{F}\left(\sqrt[q]{\mu_{2}}\right)$ and the orders of ramification of $\mathfrak{q}$ in $\mathfrak{F}\left(\sqrt[q]{\mu_{1}}\right)$ and $\mathfrak{F}\left(\sqrt[q]{\mu_{2}}\right)$ over $\mathfrak{F}$ are $\geqq a q$. In order that $\mathfrak{F}\left(\sqrt[q]{\mu_{1}}\right)$ and $\mathfrak{F}\left(\sqrt[q]{\mu_{2}}\right)$ have corresponding residue systems $\bmod \mathfrak{q}^{a q}=\mathfrak{\Omega}^{a}$ it is necessary and sufficient that the following congruences be solvable in $\mathfrak{F}$ :

$$
\begin{aligned}
& \sum_{\begin{array}{c}
e_{0}+e_{1}+\cdots+e_{q-1}=q \\
e_{1}+2 e_{2}+\cdots+(q-1) e_{q-1}=m q+i
\end{array}} \frac{q !}{e_{0} ! e_{1} ! \cdots e_{q-1} !} \alpha_{0}^{e_{0}} \alpha_{1}^{e_{1}} \cdots \alpha_{q-1}^{e_{q-1}} \mu_{2}^{m n} \equiv 0 \quad\left(\bmod \mathfrak{D}^{a q}\right) \\
& \sum_{\substack{e_{0}+\cdots+e_{q-1}=q \\
e_{1}+2 e_{2}+\cdots+(q-1) e_{q-1}=m q}} \frac{q !}{e_{0} ! \cdots e_{q-1} !} \alpha_{0}^{e_{0}} \cdots \alpha_{q-1}^{e_{q-1}} \mu_{2}^{m} \equiv \mu_{1} \quad\left(\bmod \bigcap^{a q}\right),
\end{aligned}
$$

where $\alpha_{0}, \cdots, \alpha_{q-1}$ are integers of $\mathfrak{F}$ and $e_{0}, e_{1}, \cdots, e_{q-1}, m$ are nonnegative 
integers, and $i=1, \cdots, q-1$; and the same congruences with $\mu_{1}$ and $\mu_{2}$ interchanged.

Proof. Since the orders of ramification of $q$ in $\mathfrak{F}\left(\sqrt[q]{\mu_{j}}\right)$ over $\mathfrak{F}$ are $\geqq a q$ for $j=1,2$, then either $q / \mu_{j}$ is exactly divisible by $q$ or $\sqrt[q]{\mu_{j}}$ is prime to $\mathfrak{q}$ and there exists an integer $\xi_{j}$ of $\mathfrak{F}$ such that $\sqrt[q]{\mu_{j}}-\xi_{j}$ is exactly divisible by $\mathfrak{q}$. In either case $1, \sqrt[q]{ } \mu_{j}, \cdots, q / \mu_{j}^{n-1}$ form a basis for the residue system $\bmod \mathfrak{q}^{n}, n$ a given positive integer.

If $\mathfrak{F}\left(\sqrt[q]{\mu_{1}}\right)$ and $\mathfrak{F}\left(\sqrt[q]{\mu_{2}}\right)$ have corresponding residue systems mod $q^{a q}$ we have

1.) $\sqrt[q]{\mu_{1}} \equiv \alpha_{0}+\alpha_{1} \sqrt[q]{\mu_{2}}+\cdots+\alpha_{q-1} \sqrt[q]{\mu_{2}^{q-1}}\left(\bmod \mathfrak{\Omega}^{a}\right)$

2.) $\quad \mu_{1} \equiv\left(\alpha_{\jmath}+\alpha_{1} \sqrt[q]{\mu_{2}}+\cdots+\alpha_{q-1} \sqrt[q]{\mu_{2}^{q-1}}\right)^{q} \quad\left(\bmod \mathfrak{\Omega}^{a q}\right)$

and the congruences of the theorem follow.

Conversely if the congruences of the theorem are valid then 2.) is valid and 1.) follows. Interchanging the roles of $\mu_{1}$ and $\mu_{2}$, the converse follows.

THEOREM 17. If $\mathfrak{F}=R(\zeta), q=3$, and $\mathfrak{F}\left(\sqrt[q]{\mu_{1}}\right)$ and $\mathfrak{F}\left(\sqrt[q]{\mu_{2}}\right)$ have corresponding residue systems $\bmod (1-\zeta)$, then either $\mu_{1} \equiv \alpha^{3} \mu_{2}^{\epsilon}(\bmod 3(1-\zeta))$ where $\alpha$ is in $R(\zeta)$ and $\varepsilon=1$ or 2 , or $\mu_{1} \equiv \mu_{2} \equiv 0(\bmod (1-\zeta))$.

Proof. In $R(\zeta)$ the ideal $(1-\zeta)$ is a prime ideal, that is, $(1-\zeta)=\Omega$. Since $\mathfrak{F}\left(\sqrt[q]{\mu_{1}}\right)$ and $\mathfrak{F}\left(\sqrt[q]{\mu_{2}}\right)$ have corresponding residue systems mod $(1-\zeta)$ we have $(1-\zeta)=q^{3}$, and the orders of ramification of $q$ in $\mathfrak{F}\left(\sqrt[q]{\mu_{1}}\right), \mathfrak{F}\left(\sqrt[q]{\mu_{2}}\right)$ over $\mathfrak{F}$ are $\geqq 3$, and hence either 3 or 4 . In either case $1, \sqrt[3]{\mu_{j}}, \sqrt[3]{\mu_{j}^{2}}$ form a basis for the residue system $\bmod (1-\zeta)$ in $\mathfrak{F}\left(\sqrt[q]{\mu_{j}}\right)$ for $j=1,2$.

Since $\mathfrak{F}\left(\sqrt[q]{\mu_{1}}\right)$ and $\mathfrak{F}\left(\sqrt[q]{\mu_{2}}\right)$ have corresponding residue systems mod $(1-\zeta)$, we have

$$
\begin{aligned}
\sqrt[3]{\mu_{1}} & \equiv \alpha_{0}+\alpha_{1} \sqrt[3]{\mu_{2}}+\alpha_{2} V^{3} \mu_{2}^{2} \quad(\bmod (1-\zeta)) \\
\mu_{1} & \equiv \alpha_{0}^{3}+\alpha_{1}^{3} \mu_{2}+\alpha_{2}^{3} \mu_{2}^{2}+3 P\left(\sqrt[3]{\mu_{2}}\right) \quad(\bmod 3(1-\zeta))
\end{aligned}
$$

where $P(x)$ is a polynomial with coefficients in $R(\zeta)$. It follows that $P\left(\sqrt[3]{\mu_{2}}\right)$ is congruent to a number in $R(\zeta) \bmod (1-\zeta)$, and the coefficients of $\sqrt[3]{\mu_{2}}$ and $\sqrt[3]{\mu_{2}^{2}}$ in $P\left(\sqrt[3]{\mu_{2}}\right)$ must vanish $\bmod (1-\zeta)$. Thus

$$
\alpha_{0}^{2} \alpha_{1}+\alpha_{0} \alpha_{2}^{2} \mu_{2}+\alpha_{1}^{2} \alpha_{2} \mu_{2} \equiv 0 \quad(\bmod (1-\zeta))
$$




$$
\alpha_{0} \alpha_{1}^{2}+\alpha_{1} \alpha_{2}^{2} \mu_{2}+\alpha_{0}^{2} \alpha_{2} \equiv 0 \quad(\bmod (1-\zeta)) .
$$

By considering two cases, $\mu_{2} \equiv 0(\bmod (1-\zeta))$ and $\mu_{2} \neq 0(\bmod (1-\zeta))$, the conclusion of the theorem follows from the last two congruences.

\section{REFERENCE}

1. E. Hecke, Theorie der Algebraischen Zahlen, Leipzig, 1923.

Louisiana State University and Ohio State University 


\section{PACIFIC JOURNAL OF MATHEMATICS}

EDITORS

\author{
H. L. Royden \\ Stanford University \\ Stanford, California \\ E. Hewite \\ University of Washington \\ Seattle 5 , Washington
}

\author{
R. P. Dilworth \\ California Institute of Technology \\ Pasadena 4, California
}

\author{
A. Horn* \\ University of California \\ Los Angeles 24, California
}

\section{ASSOCIATE EDITORS}
E. F. BECKENBACH
M. HALL
M. S. KNEBELMAN
J. J. STOKER
C. E. BURGESS
P. R. HALMOS
I. NIVEN
V. GANAPATHY IYER
T. G. OSTROM
G. SZEKERES
H. BUSEMANN
R. D. JAMES
M. M. SCHIFFER
F. WOLF
H. FEDERER

\section{SPONSORS}

\author{
UNIVERSITY OF BRITISH COLUMBIA \\ CALIFORNIA INSTITUTE OF TECHNOLOGY \\ UNIVERSITY OF CALIFORNIA \\ CALIFORNIA RESEARCH CORPORATION \\ MONTANA STATE UNIVERSITY \\ UNIVERSITY OF NEVADA \\ OREGON STATE COLLEGE \\ UNIVERSITY OF OREGON \\ UNIVERSITY OF SOUTHERN CALIFORNIA
}

\author{
STANFORD UNIVERSITY \\ UNIVERSITY OF UTAH \\ WASHINGTON STATE COLLEGE \\ UNIVERSITY OF WASHINGTON \\ AMERICAN MATHEMATICAL SOCIETY \\ HUGHES AIRCRAFT COMPANY
}

Mathematical papers intended for publication in the Pacific Journal of Mathematics should be typewritten (double spaced), and the author should keep a complete copy. Manuscripts may be sent to any of the editors. Manuscripts intended for the outgoing editors should be sent to their successors. All other communications to the editors should be addressed to the managing editor, Alfred Horn at the University of California, Los Angeles 24, California.

50 reprints of each article are furnished free of charge; additional copies may be obtained at cost in multiples of 50 .

The Pacific Journal of Mathematics is published quarterly, in March, June, September, and December. The price per volume (4 numbers) is $\$ 12.00$; single issues, $\$ 3.50$. Back numbers are available. Special price to individual faculty members of supporting institutions and to individual members of the American Mathematical Society: $\$ 4.00$ per volume; single issues, $\$ 1.25$.

Subscriptions, orders for back numbers, and changes of address should be sent to Pacific Journal of Mathematics, c/o University of California Press, Berkeley 4, California.

Printed at Kokusai Bunken Insatsusha (International Academic Printing Co., Ltd.), No. 10, 1-chome, Fujimi-cho, Chiyoda-ku, Tokyo, Japan.

* During the absence of E. G. Straus.

PUBLISHED BY PACIFIC JOURNAL OF MATHEMATICS, A NON-PROFIT CORPORATION COPYRIGHT 1956 BY PACIFIC JOURNAL OF MATHEMATICS 


\section{Pacific Journal of Mathematics}

\section{Vol. 6, No. $2 \quad$ December, 1956}

Louis Auslander, Remark on the use of forms in variational calculations .......................................... 209

Hubert Spence Butts, Jr. and Henry B. Mann, Corresponding residue systems in algebraic number fields ........................ 211

L. Carlitz and John Herbert Hodges, Distribution of matrices in a finite field............................................

Paul Civin and Bertram Yood, Invariant functionals ............... 231

David James Dickinson, Henry Pollak and G. H. Wannier, On a class of polynomials orthogonal over a denumerable set .................

Bernard Friedman and Luna Mishoe, Eigenfunction expansions associated with a non-self-adjoint differential equation ....................

Luna Mishoe and G. C. Ford, On the uniform convergence of a certain eigenfunction series .............................. 271

John W. Green, Mean values of harmonic functions on homothetic curves...........................................

Charles John August Halberg, Jr. and Angus E. Taylor, On the spectra of linked operators .....................................

Chuan Chih Hsiung, Some integral formulas for closed hypersurfaces in Riemannian space ................................... 291

Norman D. Lane, Differentiable points of arcs in conformal n-space 301

Louis F. McAuley, A relation between perfect separability, completeness, and normality in semi-metric spaces

G. Power and D. L. Scott-Hutton, The slow shearing motion of a liquid past a semi-infinite plane .............................

A. C. Schaeffer, Entire functions

Edward Silverman, An intrinsic inequality for Lebesgue area...

Choy-Tak Taam, Asymptotic relations between systems of differential equations.

Ti Yen, Quotient algebra of a finite $A W^{*}$-algebra ... 